\title{
CORRESPONDENCE
}

\section{Comment on: Effect of a nicotine-free inhalator as part of a smoking cessation programme}

\section{To the Editors:}

The study by CAPONNETTO et al. [1] in the November 2011 issue of the European Respiratory Journal addresses the behavioural part of smoking. The purpose of this comment is to offer a constructive review of the research and suggestions for further research.

In this study at a smokers' clinic, 120 smokers were given pharmacological treatment at their choice but were also randomised to receive a cigarette-like nicotine-free inhalator loaded with a sponge soaked in naturally extracted herbal aroma oil.

The smoking cessation results showed no main effect of this inhalator but quite a pronounced effect of the inhalator in a group with a supposedly high degree of behaviour dependence to smoking. The behaviour dependence was measured according to the Glover-Nilsson Smoking Behavioral Questionnaire (GN-SBQ) scale. This questionnaire asks how important the behavioural part of smoking is [2].

In the intention-to-treat analysis at 24-week follow-up, $28 \%$ and $33 \%$ were not smoking in the reference (no inhalator) and inhalator groups, respectively. In the group of subjects with high behaviour dependence ( $>22$ points on the GN-SBQ), the corresponding cessation rates were 19\% and 67\% $(\mathrm{p}<0.007)$. This finding is surprisingly strong, given everything we know about predictors for smoking cessation outcome, particularly since the GN-SBQ so far has not shown any acceptable validity. Only one study is cited in support of the scale [2], which does not prove validity.

Based upon the findings in this study, several questions emerge that may benefit future replications of this study. First, how was the cut-off of 22 points on the GN-SBQ chosen? Was it an ad hoc decision? Secondly, how were the pharmacological treatments distributed between the groups? Was there an interaction of pharmacological treatment and inhalator use? Thirdly, treatments usually have their best effect early on in the trials. In this trial, the effect of the inhalator was stronger at week 24 than at week 4 . Did subjects use more of the inhalator after week 4? How did the use pattern look over time? Fourthly, it is one thing to accept that the inhalator could be effective for those with high behaviour dependence but somewhat difficult to understand that it should negatively influence outcome in the low behaviour dependence group. One would think that all daily smokers, smoking a mean of 25 cigarettes $\cdot$ day $^{-1}$ over a mean period of $29 \mathrm{yrs}$, would be behaviourally dependent. How did the use pattern of the inhalator vary between the two treatment groups? Finally, it would be of more general interest to know the correlation between the GN-SBQ and the Fagerström Test for Nicotine Dependence to clarify whether the two types of dependencies are independent of each other or correlated. I would assume that a smoker highly dependent on nicotine would also tend to be more behaviourally dependent.

In conclusion, smokers differ in dependence profiles and the problems they face when stopping smoking. Better diagnosis of the smokers, which this study aims for, could ultimately lead to tailoring treatments and boosting efficacy [3]. This first study was small and needs to be replicated with larger samples and by other investigators. Until then, we have to be cautious, but some further analysis of the data from this study as suggested here could at least shed some more light on the matter.

\section{K. Fagerström \\ Fagerström Consulting, Kågeröd, Sweden.}

Correspondence: K. Fagerström, Fagerström Consulting, Jordkull 3670, 26878 Kågeröd, Sweden. E-mail: karl.fagerstrom@ swipnet.se

Statement of Interest: None declared.

\section{REFERENCES}

1 Caponnetto P, Cibella F, Mancuso S, et al. Effect of a nicotine-free inhalator as part of a smoking-cessation programme. Eur Respir J 2011; 38: 1005-1011.

2 Glover ED, Nilsson F, Westin A, et al. Developmental history of the Glover-Nilsson smoking behavioral questionnaire. Am J Health Behav 2005; 29: 443-455.

3 Quaak M, van Schayck CP, Knaapen AM, et al. Genetic variation as a predictor of smoking cessation success. A promising preventive and intervention tool for chronic respiratory diseases? Eur Respir J 2009; 33: $468-480$.

DOI: $10.1183 / 09031936.00116111$

\section{To the Editors:}

In their recent publication, CAPONNETTO et al. [1] described the effect of randomised nicotine-free inhalator use during a smoking cessation intervention with 24 weeks of follow-up. Interestingly, the effect differed in direction according to the degree of nicotine dependency assessed by the Glover-Nilsson Smoking Behavioral Questionnaire, with a reduced smoking quit rate among low-rate dependency persons and an increased quit rate among high-rate dependency persons. 